\title{
Finite-time synchronization problem of a class of discontinuous Cohen-Grossberg neural networks with mixed delays via new switching design
}

\section{Zhaohong Xiang ${ }^{1 *}$ (D) and Runjie Li ${ }^{2}$}

"Correspondence:

xp0745@ccsu.edu.cn

'Department of Mathematics and

Computer Science, Changsha University, Changsha 410022, China Full list of author information is available at the end of the article

\section{Springer}

\begin{abstract}
This paper investigates a class of generalized Cohen-Grossberg neural networks (CGNNs) with discontinuous activations and mixed delays. Based on the nonsmooth analysis theory, the drive-response concept, differential inclusions theory, we give several basic assumptions to gain the finite-time synchronization issue of CGNNs. Sufficient conditions are provided without the boundedness or monotonicity of discontinuous activation functions. Moreover, one can estimate the settling time's upper bounds of the system. At last, two numerical examples and their simulations are given to further show the benefits of the obtained control approach.
\end{abstract}

Keywords: Finite-time synchronization; Cohen-Grossberg neural networks; Discontinuous activations; Mixed delays

\section{Introduction}

Recently, the research of neural networks with discontinuous activation functions has gradually attracted the attention of many researchers, including systems oscillating under earthquake, power circuits, chaos phenomenon, and dry friction (see [1-4]). Because the non-Lipschitz phenomenon has many special advantages, the emergence of nonsmooth has greatly improved the research of neural networks. In addition, the analysis of neural networks with discontinuous activations is accompanied by many interesting practical phenomena to explore important dynamic behavior characteristics. This arouses researchers' interest in the generalized neural networks by using discontinuous activation functions [5-8].

In 1983, Cohen and Grossberg firstly introduced the CGNNs system, which was a useful recurrent neural networks system, including evolutionary theory, population biology, neurobiology [9]. After that, a large number of results have emerged [10-17] such as the existence, dissipation, and exponential stability of the CGNNs model. However, there are few works on discontinuous CGNNs system with mixed delays. In [18-20], the authors investigated the exponential stability and exponential synchronization of a class of CGNNs.

(c) The Author(s) 2021. This article is licensed under a Creative Commons Attribution 4.0 International License, which permits use sharing, adaptation, distribution and reproduction in any medium or format, as long as you give appropriate credit to the original author(s) and the source, provide a link to the Creative Commons licence, and indicate if changes were made. The images or other third party material in this article are included in the article's Creative Commons licence, unless indicated otherwise in a credit line to the material. If material is not included in the article's Creative Commons licence and your intended use is not permitted by statutory regulation or exceeds the permitted use, you will need to obtain permission directly from the copyright holder. To view a copy of this licence, visit http://creativecommons.org/licenses/by/4.0/. 
Abdurahman and his team in [21] studied the exponential lag synchronization for both discrete time-delays and distributed delays CGNNs. It worthy to know that, in 2003, Forti introduced the global stability of a discontinuous right-hand side neural network system via the framework of the theory of Filippov differential inclusions [22, 23]. In [24] they pointed out that the sliding mode method was used to solve constrained optimization problems, because high-gain neuron activations were often encountered in the neural networks system. In [25], the authors analyzed the fixed-time synchronization of a class of discontinuous fuzzy inertial neural networks with time-varying delays based on the new improved fixed-time stability lemmas.

As we know, the synchronization phenomenon has been widely used in software engineering, ecological structure, security storage, information processing system, and many other fields, which gets a lot of social attention. There are some micro motions from the view of the mathematical model, which we call qualitative or stability problems. Meanwhile, the researchers pay attention to the macroscopic topology based on the synchronization problem. The literature [26] was the first paper to consider finite-time control of discontinuous chaotic systems, and [27] studied the finite-time synchronization of timedelayed neural networks. There were many classifications of synchronization, such as anti-synchronization [28, 29], exponential synchronization [30], robust synchronization [31], chaos synchronization [32], and so on. The synchronization technologies mentioned above have many defects in a real practical environment. For instance, the existence results of the above synchronization usually are guaranteed over the infinite horizon. In addition, when the finite initial value is required and the control accuracy has great influence on the system, it is always difficult to estimate. Moreover, even if stringent convergence time is given, the neural networks model may not be available in a real experimental environment. For the sake of convergence time, one needs to propose a concept named finite-time synchronization, which means that the settling time function of any finite initial value is bounded. Song and his team provided a novel and effective techniques method in [33], then in [34] they investigated the finite-time synchronization problem of a class of discontinuous neural networks with nonlinear coupling and mixed delays. Peng and his team in [35] investigated the finite-time synchronization control methodology for the CGNNs system. Yang in [36] verified that the considered neural networks can gain the synchronization in a finite time. In [37], the authors ensured that the target model realized the finite-time synchronization goal of the coupled neural networks. In [38], the author solved the challenging issues in the field of finite-time synchronization of the cellular neural networks.

Motivated by the aforementioned works on finite-time synchronization of CGNNs system, this paper aims to realize the finite-time synchronization issue for the considered system CGNNs. Our main contributions of this paper include the following three aspects.

- The CGNNs discussed in this brief are state-dependent discontinuous systems; based on the properties of differential inclusion and set-analysis theory, the drive-response CGNNs can be transformed into a synchronization error system. Theoretical analysis can be extended to other fields.

- When both mixed delays and discontinuities exist in the dynamical CGNNs, how to deal with the discrepancy within the scope of the Filippov solutions of the drive system and the response system? 
- Because the system has special discontinuous characteristics, in order to shorten the settling time of the drive-response CGNNs system, the more ingenious switching controller should be devised.

\section{Model description and some basic definitions}

This section considers a general class of discontinuous CGNNs with mixed delays. Based on the previous works [39-45], one can describe the model by the following equation:

$$
\begin{aligned}
\frac{\mathrm{d} \pi_{i}(t)}{\mathrm{d} t}= & -\varpi_{i}\left(\pi_{i}(t)\right)\left[a_{i}\left(\pi_{i}(t)\right)-\sum_{j=1}^{n} b_{i j}(t) f_{j}\left(\pi_{j}(t)\right)-\sum_{j=1}^{n} c_{i j}(t) f_{j}\left(\pi_{j}\left(t-\tau_{i j}(t)\right)\right)\right. \\
& \left.-\sum_{j=1}^{n} \int_{0}^{+\infty} K_{i j}(t, s) f_{j}\left(\pi_{j}(s)\right) \mathrm{d} s-I_{i}(t)\right], \quad i=1,2, \ldots, n,
\end{aligned}
$$

the state vector is $\pi(t)=\left(\pi_{1}(t), \pi_{2}(t), \ldots, \pi_{n}(t)\right)^{T} \in \mathbb{R} ; \varpi_{i}(\cdot)$ is the amplification function of the system; $a_{i}(\cdot)$ is the function with proper behavior; $\tau_{i j}(t)$ and $K_{i j}:[0, \infty) \rightarrow[0, \infty)$ denote the discrete delay and the distributed delay, respectively; $b_{i j}(t)$ and $c_{i j}(t)$ are the connection strength and the delayed feedbacks of two different neurons; $f_{j}(\cdot)$ is the neuron input-output activation of the $i$ th neuron; $I_{i}(\cdot)$ is an input signal function of the external factors;

The neuron activation functions $f_{j}(\cdot)$ in the above model satisfy the following conditions:

(H1) $f_{i}: \mathbb{R} \rightarrow \mathbb{R}$ is discontinuous on a countable set of isolate point $\left\{\rho_{k}^{i}\right\}$ for each $i=1,2, \ldots, n$.

(H2) There are two nonnegative constants $L_{i}$ and $h_{i}$ that satisfy the following inequality:

$$
\left\|\mathbb{F}\left[f_{i}(x)-f_{i}(y)\right]\right\|=\sup _{\zeta_{i} \in \mathbb{F}\left[f_{i}(x)-f_{i}(y)\right]}\left\|\zeta_{i}\right\| \leq L_{i}\|x-y\|+h_{i}, \quad i=1,2, \ldots, n
$$

where $\mathbb{F}\left(f_{i}(x)\right)=K\left[f_{i}(x)\right]=\left[\min \left\{f_{i}\left(x_{i}^{-}\right), f_{i}\left(x_{i}^{+}\right)\right\}, \max \left\{f_{i}\left(x_{i}^{-}\right), f_{i}\left(x_{i}^{+}\right)\right\}\right]$.

(H3) There exist nonnegative constants $K_{i j}$ satisfying

$$
\int_{0}^{+\infty} K_{i j}(\cdot) \mathrm{d} s \leq K_{i j}, \quad i, j=1,2 \ldots, n
$$

For every $i, j=1,2, \ldots, n$, we assume that $a_{i}(t), b_{i j}(t), c_{i j}(t), \tau_{i j}(t)$ are continuous $\omega$ periodic functions and $I_{i}(t)$ are almost periodic functions; $0 \leq \tau_{i j}(t) \leq \tau_{i j}, \dot{\tau}_{i j}(t) \leq \sigma_{i j}<$ $1 ; \varpi_{i}(\cdot)$ is continuous and $0<\underline{\omega} \leq \varpi_{i}(\cdot) \leq \bar{\omega} ; \dot{a}_{i}(\cdot) \geq a_{i}$, where $\tau_{i j}, \sigma_{i j}, \underline{\omega}$, $\bar{\omega}$, and $a_{i}$ are nonnegative constants. Moreover, we denote $a^{\max }=\max _{1 \leq i \leq n} \sup _{t \in \mathbb{R}}\left|a_{i}(t)\right|, b^{\max }=$ $\max _{1 \leq i \leq n, 1 \leq j \leq n} \sup _{t \in \mathbb{R}}\left|b_{i j}(t)\right|, c^{\max }=\max _{1 \leq i \leq n, 1 \leq j \leq n} \sup _{t \in \mathbb{R}}\left|c_{i j}(t)\right|$.

Choose a transformation function $\Phi_{i}^{-1}(\cdot)$, which satisfies

$$
\frac{\mathrm{d}}{\mathrm{d} u}\left(\Phi_{i}^{-1}(u)\right)=\frac{1}{\varpi_{i}(u)}
$$

From the above discussion we know that $\frac{1}{\bar{w}_{i}(u)}>0$, which yields that $\Phi_{i}^{-1}(\cdot)$ is strictly monotone increasing, then the inverse function of $\Phi_{i}^{-1}(\cdot)$ exists, we denote $\left(\Phi_{i}^{-1}(\cdot)\right)^{-1}=$ 
$\Phi_{i}(\cdot)$. Let $x_{i}(t)=\Phi_{i}^{-1}\left(\pi_{i}(t)\right)$, one can have $\pi_{i}(t)=\Phi_{i}(x(t))$ and $\frac{\mathrm{d} x_{i}(t)}{\mathrm{d} t}=\frac{\mathrm{d} \Phi_{i}^{-1}\left(\pi_{i}(t)\right)}{\mathrm{d} \pi_{i}(t)} \dot{\pi}_{i}(t)=$ $\frac{1}{\varpi_{i}\left(\pi_{i}(t)\right)} \dot{\pi}_{i}(t)$, then we can obtain that

$$
\begin{aligned}
\frac{\mathrm{d} x_{i}(t)}{\mathrm{d} t}= & -a_{i}\left(\Phi_{i}\left(x_{i}(t)\right)\right)+\sum_{j=1}^{n} b_{i j}(t) f_{j}\left(\Phi_{i}\left(x_{i}(t)\right)\right)+\sum_{j=1}^{n} c_{i j}(t) f_{j}\left(\Phi_{i}\left(x_{i}\left(t-\tau_{i j}(t)\right)\right)\right) \\
& +\sum_{j=1}^{n} \int_{0}^{+\infty} K_{i j}(t, s) f_{j}\left(\Phi_{i}\left(x_{i}(s)\right)\right) \mathrm{d} s+I_{i}(t), \quad i=1,2, \ldots, n .
\end{aligned}
$$

Obviously, in the framework of differential inclusions, system (2) can be rewritten as follows:

$$
\begin{aligned}
\frac{\mathrm{d} x_{i}(t)}{\mathrm{d} t} \in & -a_{i}\left(\Phi_{i}\left(x_{i}(t)\right)\right)+\sum_{j=1}^{n} b_{i j}(t) K\left[f_{j}\left(\Phi_{i}\left(x_{i}(t)\right)\right)\right]+\sum_{j=1}^{n} c_{i j}(t) K\left[f_{j}\left(\Phi_{i}\left(x_{i}\left(t-\tau_{i j}(t)\right)\right)\right)\right] \\
& +\sum_{j=1}^{n} \int_{0}^{+\infty} K_{i j}(t, s) K\left[f_{j}\left(\Phi_{i}\left(x_{i}(s)\right)\right)\right] \mathrm{d} s+I_{i}(t) .
\end{aligned}
$$

For any compact interval of $[0, \tau)$, the vector function $x=\left(x_{1}, x_{2}, \ldots, x_{n}\right)^{T}$ is continuous and absolutely continuous. According to the Filippov framework, one can find a measurable function $\gamma=\left(\gamma_{1}, \gamma_{2}, \ldots, \gamma_{n}\right)^{T}:(-\infty, \tau) \rightarrow \mathbb{R}^{n}$ such that $\gamma_{i}(t) \in K\left[f_{i}\left(\Phi_{i}\left(x_{i}(t)\right)\right)\right]$, then one can obtain that $x$ is a state solution of CGNNs and

$$
\begin{aligned}
\frac{\mathrm{d} x_{i}(t)}{\mathrm{d} t}= & -a_{i}\left(\Phi_{i}\left(x_{i}(t)\right)\right)+\sum_{j=1}^{n} b_{i j}(t) \gamma_{j}(t)+\sum_{j=1}^{n} c_{i j}(t) \gamma_{j}(t-\tau) \\
& +\sum_{j=1}^{n} \int_{0}^{+\infty} K_{i j}(t, s) \gamma_{j}(s) \mathrm{d} s+I_{i}(t) .
\end{aligned}
$$

Through the above discussion, consider CGNNs system (1) as the drive system. By giving the initial value of CGNNs $\phi(s)=\left(\phi_{1}(s), \phi_{2}(s), \ldots, \phi_{n}(s)\right)^{T}$, we can obtain the corresponding response system as follows:

$$
\begin{aligned}
\frac{\mathrm{d} \xi_{i}(t)}{\mathrm{d} t}= & -\varpi_{i}\left(\xi_{i}(t)\right)\left[a_{i}\left(\xi_{i}(t)\right)-\sum_{j=1}^{n} b_{i j}(t) f_{j}\left(\xi_{j}(t)\right)-\sum_{j=1}^{n} c_{i j}(t) f_{j}\left(\xi_{j}\left(t-\tau_{i j}(t)\right)\right)\right. \\
& \left.-\sum_{j=1}^{n} \int_{0}^{+\infty} K_{i j}(t, s) f_{j}\left(\xi_{j}(s)\right) \mathrm{d} s-I_{i}(t)\right]+u_{i}(t), \quad i=1,2, \ldots, n,
\end{aligned}
$$

where $u_{i}(t)$ is the appropriate controller.

Similarly, let $y_{i}(t)=\Phi_{i}^{-1}\left(\xi_{i}(t)\right), i=1,2, \ldots, n$, we can derive that

$$
\begin{aligned}
\frac{\mathrm{d} y_{i}(t)}{\mathrm{d} t}= & -a_{i}\left(\Phi_{i}\left(y_{i}(t)\right)\right)+\sum_{j=1}^{n} b_{i j}(t) \widetilde{\gamma}_{j}(t)+\sum_{j=1}^{n} c_{i j}(t) \widetilde{\gamma}_{j}(t-\tau) \\
& +\sum_{j=1}^{n} \int_{0}^{+\infty} K_{i j}(t, s) \widetilde{\gamma}_{j}(s) \mathrm{d} s+I_{i}(t)+\frac{u_{i}(t)}{\varpi_{i}\left(\Phi_{i}\left(y_{i}(t)\right)\right)},
\end{aligned}
$$

where $\tilde{\gamma}_{i}(t) \in K\left[f_{i}\left(\Phi_{i}\left(y_{i}(t)\right)\right)\right]$ and $\tilde{\gamma}(t)=\left(\gamma_{1}(t), \gamma_{2}(t), \ldots, \gamma_{n}(t)\right)^{T}$. 
Lemma 2.1 (See [23, 39]) If $V(y(t)): \mathbb{R}^{n} \times \mathbb{R}$ is a C-regular function, for any compact interval of $[0,+\infty), y(t):[0,+\infty) \rightarrow \mathbb{R}^{n}$ is an absolutely continuous function. For a continuous function $\Upsilon:(0, \infty) \rightarrow \mathbb{R}$ with $\Upsilon(\varrho)>0$ for $\varrho \in(0,+\infty)$, if it satisfies that

$$
\frac{d V(t)}{d t} \leq-\Upsilon(V(t)) \text { for a.e. } t \geq 0
$$

and

$$
\int_{0}^{V(0)} \frac{1}{\Upsilon(\varrho)}=t^{*}<+\infty
$$

then we have $V(t)=0$ for $t \geq t^{*}$; especially, we have:

(1) If $\Upsilon=K_{1} \varrho+K_{2} \varrho^{\mu}$ for all $\varrho>0$, where $\mu \in(0,1)$ and $K_{1}, K_{2}>0$, then one can estimate the settling time as

$$
t^{*}=\frac{1}{K_{1}(1-\mu)} \ln \frac{K_{1} V^{1-\mu}(0)+K_{2}}{K_{2}} .
$$

(2) If $\Upsilon(\varrho)=K \varrho^{\mu}$ and $K>0$, then one can estimate the settling time as

$$
t^{*}=\frac{V^{1-\mu}(0)}{K(1-\mu)}
$$

Remark 2.2 Since $\Phi(\cdot)$ is strictly monotone increasing and $a_{i}\left(\Phi_{i}(t)\right)$ is an abstract function which contains linear functions as special cases. In other words, we can express $a_{i}\left(\Phi_{i}(t)\right)$ as a common function $-D_{i}(t) \Phi_{i}(t)$. Compared with the existing papers, our model which considers CGNNs is more general and common of previous results.

\section{Main results}

Firstly, we focus on ensuring the finite-time synchronization issue between the above response model (4) and the drive model (1). Let $e_{i}(t)=y_{i}(t)-x_{i}(t), i=1,2, \ldots, n$, one can obtain

$$
\begin{aligned}
\frac{\mathrm{d} e_{i}(t)}{\mathrm{d} t}= & -\left(a_{i}\left(\Phi_{i}\left(y_{i}(t)\right)\right)-a_{i}\left(\Phi_{i}\left(x_{i}(t)\right)\right)\right)+\sum_{j=1}^{n} b_{i j}(t) \gamma_{j}^{*}(t)+\sum_{j=1}^{n} c_{i j}(t) \gamma_{j}^{*}(t-\tau) \\
& +\sum_{j=1}^{n} \int_{0}^{+\infty} K_{i j}(t, s) \gamma_{j}^{*}(s) \mathrm{d} s+\frac{u_{i}(t)}{\varpi_{i}\left(\Phi_{i}\left(y_{i}(t)\right)\right)},
\end{aligned}
$$

where $\gamma_{j}^{*}(t)=\tilde{\gamma}_{j}(t)-\gamma_{j}(t)$.

Then we consider the following two kinds of important controllers to achieve the finitetime synchronization issue.

Case (1). The state-feedback controller $u_{i}(t)$ :

$$
u_{i}(t)=-k_{1}\left(\pi_{i}(t)-\xi_{i}(t)\right)-k_{2} \operatorname{sign}\left(\pi_{i}(t)-\xi_{i}(t)\right)
$$

where $i=1,2, \ldots, N, k_{1}, k_{2}>0$. 
Case (2). The corresponding adaptive controller $u_{i}(t)$ s of Case (1):

$$
u_{i}(t)=-p_{i}\left(\pi_{i}(t)-\xi_{i}(t)\right)-q_{i} \operatorname{sign}\left(\pi_{i}(t)-\xi_{i}(t)\right)
$$

where $P=\operatorname{diag}\left(p_{1}, p_{2}, \ldots, p_{n}\right), Q=\operatorname{diag}\left(q_{1}, q_{2}, \ldots, q_{n}\right)$, and the controller rules of $p_{i}$ and $q_{i}$ are as follows:

$$
\begin{aligned}
& \dot{p}_{i}=e_{i}^{T}(t) \frac{\rho_{i}}{\varpi_{i}\left(y_{i}(t)\right)}\left(\pi_{i}(t)-\xi_{i}(t)\right) \text { and } \\
& \dot{q}_{i}=e_{i}^{T}(t) \frac{\varrho_{i}}{\varpi_{i}\left(y_{i}(t)\right)} \operatorname{sign}\left(\pi_{i}(t)-\xi_{i}(t)\right), \quad i=1,2, \ldots, n,
\end{aligned}
$$

$\rho_{i}$ and $\varrho_{i}$ are arbitrary positive constants.

Theorem 3.1 If conditions (H1)-(H3) are supported, the response system (4) with statefeedback controller (7) can synchronize to the corresponding drive system (1) in a finite-time if the following assumption holds:

$(\mathrm{H} 4) \underline{\omega}+\frac{k_{1} \cdot \underline{\omega}}{\bar{\omega}}>\left(b^{\max }+c^{\max }+K^{\max }\right) \cdot n L^{\max }$ and $\frac{k_{2}}{\bar{\omega}}>\left(b^{\max }+c^{\max }+K^{\max }\right) \cdot n h^{\max }$.

Proof Let

$$
V(t)=\frac{1}{2} e^{T}(t) e(t)+\frac{n}{2} \cdot L^{\max } c^{\max } \int_{t-\tau}^{t} e^{T}(s) \cdot e(s) \mathrm{d} s,
$$

where $e(t)=\left(e_{1}(t), e_{2}(t), \ldots, e_{n}(t)\right)^{T}$.

Obviously $V(t)$ is C-regular. The derivative of Lyapunov function $V(t)$ can be obtained along the error system (6) as follows:

$$
\begin{aligned}
\frac{\mathrm{d} V(t)}{\mathrm{d} t}= & e^{T}(t) \dot{e}(t)=\sum_{i=1}^{n} e_{i}^{T}(t) \dot{e}_{i}(t) \\
= & \sum_{i=1}^{n} e_{i}^{T}(t)\left(-\left(a_{i}\left(\Phi_{i}\left(y_{i}(t)\right)\right)-a_{i}\left(\Phi_{i}\left(x_{i}(t)\right)\right)\right)+\sum_{j=1}^{n} b_{i j}(t) \gamma_{j}^{*}(t)\right. \\
& +\sum_{j=1}^{n} c_{i j}(t) \gamma_{j}^{*}(t-\tau)+\sum_{j=1}^{n} \int_{0}^{+\infty} K_{i j}(t, s) \gamma_{j}^{*}(s) \mathrm{d} s \\
& \left.-\frac{k_{1}}{\varpi_{i}\left(y_{i}(t)\right)}\left(\pi_{i}(t)-\xi_{i}(t)\right)-\frac{k_{2}}{\varpi_{i}\left(y_{i}(t)\right)} \operatorname{sign}\left(\pi_{i}(t)-\xi_{i}(t)\right)\right) \\
& +\frac{n}{2} \cdot L^{\max } c^{\max } e^{T}(t) e(t)-\frac{n}{2} \cdot L^{\max } c^{\max } e^{T}(t-\tau) e(t-\tau) \\
= & -\sum_{i=1}^{n} e_{i}^{T}(t)\left(a_{i}\left(\Phi_{i}\left(y_{i}(t)\right)\right)-a_{i}\left(\Phi_{i}\left(x_{i}(t)\right)\right)\right)+\sum_{i=1}^{n} \sum_{j=1}^{n} e_{i}^{T}(t) b_{i j}(t) \gamma_{j}^{*}(t) \\
& +\sum_{i=1}^{n} \sum_{j=1}^{n} e_{i}^{T}(t) c_{i j}(t) \gamma_{j}^{*}(t-\tau)+\sum_{i=1}^{n} \sum_{j=1}^{n} e_{i}^{T}(t) \int_{0}^{+\infty} K_{i j}(t, s) \gamma_{j}^{*}(s) \mathrm{d} s \\
& -\sum_{i=1}^{n} e_{i}^{T}(t) k_{1} \frac{1}{\varpi_{i}\left(y_{i}(t)\right)}\left(\Phi_{i}\left(y_{i}(t)\right)-\Phi_{i}\left(x_{i}(t)\right)\right) \\
& \\
&
\end{aligned}
$$




$$
\begin{aligned}
& \left.-\sum_{i=1}^{n} e_{i}^{T}(t) k_{2} \frac{1}{\varpi_{i}\left(y_{i}(t)\right)} \operatorname{sign}\left[\Phi_{i}\left(y_{i}(t)\right)\right)-\Phi_{i}\left(x_{i}(t)\right)\right] \\
& +\frac{n}{2} \cdot L^{\max } c^{\max } e^{T}(t) e(t)-\frac{n}{2} \cdot L^{\max } c^{\max } e^{T}(t-\tau) e(t-\tau) .
\end{aligned}
$$

Based on the definition of function $\Phi(\cdot)$ and generalized mean value theorem, one can have

$$
-\sum_{i=1}^{n} e_{i}^{T}(t)\left(a_{i}\left(\Phi_{i}\left(y_{i}(t)\right)\right)-a_{i}\left(\Phi_{i}\left(x_{i}(t)\right)\right)\right) \leq-\sum_{i=1}^{n} e_{i}^{T}(t) \underline{\omega} e_{i}(t) .
$$

From assumptions $(\mathrm{H} 1)-(\mathrm{H} 2)$, we can obtain that

$$
\begin{aligned}
\sum_{i=1}^{n} \sum_{j=1}^{n} e_{i}^{T}(t) b_{i j}(t) \gamma_{j}^{*}(t) & \leq \sum_{i=1}^{n} \sum_{j=1}^{n}\left|e_{i}^{T}(t)\right| \cdot\left|b_{i j}(t)\right| \cdot\left|\gamma_{j}^{*}(t)\right| \\
& \leq \sum_{i=1}^{n} \sum_{j=1}^{n}\left|e_{i}^{T}(t)\right| \cdot\left|b_{i j}(t)\right| \cdot\left(L_{j}\left|e_{j}(t)\right|+h_{j}\right) \\
& \leq n b^{\max } L^{\max } \sum_{i=1}^{n} e_{i}^{T}(t) e_{i}(t)+n b^{\max } h^{\max } \sum_{i=1}^{n} \sum_{k=1}^{n}\left|e_{i k}(t)\right|
\end{aligned}
$$

and

$$
\begin{aligned}
\sum_{i=1}^{n} \sum_{j=1}^{n} e_{i}^{T}(t) c_{i j}(t) \gamma_{j}^{*}(t-\tau) \leq & \sum_{i=1}^{n} \sum_{j=1}^{n}\left|e_{i}^{T}(t)\right| \cdot\left|c_{i j}(t)\right| \cdot\left|\gamma_{j}^{*}(t-\tau)\right| \\
\leq & \sum_{i=1}^{n} \sum_{j=1}^{n}\left|e_{i}^{T}(t)\right| \cdot\left|c_{i j}(t)\right| \cdot\left(L_{j}\left|e_{j}(t-\tau)\right|+h_{j}\right) \\
\leq & c^{\max } L^{\max } \sum_{i=1}^{n} \sum_{j=1}^{n} e_{i}^{T}(t) e_{j}(t-\tau)+n c^{\max } h^{\max } \sum_{i=1}^{n} \sum_{i=1}^{n}\left|e_{i k}(t)\right| \\
\leq & c^{\max } L^{\max }\left(\frac{n}{2} \sum_{i=1}^{n} e_{i}^{T}(t) e_{i}(t)+\frac{n}{2} \sum_{j=1}^{n} e_{j}^{T}(t-\tau) e_{j}(t-\tau)\right) \\
& +n c^{\max } h^{\max } \sum_{i=1}^{n} \sum_{k=1}^{n}\left|e_{i k}(t)\right| \cdot
\end{aligned}
$$

From condition (H3), similarly we have

$$
\begin{aligned}
\sum_{i=1}^{n} \sum_{j=1}^{n} e_{i}^{T}(t) \int_{0}^{+\infty} K_{i j}(t, s) \gamma_{j}^{*}(s) \mathrm{d} s \leq & \sum_{i=1}^{n} \sum_{j=1}^{n} K_{i j} e_{i}^{T}(t) \gamma_{j}^{*}(s) \\
\leq & n K^{\max } L^{\max } \sum_{i=1}^{n} e_{i}^{T}(t) e_{i}(t) \\
& +n K^{\max } h^{\max } \sum_{i=1}^{n} \sum_{k=1}^{n}\left|e_{i k}(t)\right| .
\end{aligned}
$$


Moreover, because $\Phi_{i}(\cdot)$ is strictly monotone increasing, which implies that $\operatorname{sign}\left(\Phi_{i}\left(y_{i}(t)\right)-\Phi_{i}\left(x_{i}(t)\right)\right)=\operatorname{sign}\left(y_{i}(t)-x_{i}(t)\right)=\operatorname{sign} e_{i}(t)$, then we obtain

$$
\begin{aligned}
& -\sum_{i=1}^{n} e_{i}^{T}(t) k_{1} \frac{1}{\varpi_{i}\left(y_{i}(t)\right)}\left(\Phi_{i}\left(y_{i}(t)\right)-\Phi_{i}\left(x_{i}(t)\right)\right) \leq-\sum_{i=1}^{n} \frac{k_{1} \cdot \underline{\omega}}{\bar{\omega}} e_{i}^{T}(t) e_{i}(t), \\
& -\sum_{i=1}^{n} e_{i}^{T}(t) k_{2} \frac{1}{\varpi_{i}\left(y_{i}(t)\right)} \operatorname{sign}\left[\Phi_{i}\left(y_{i}(t)\right)-\Phi_{i}\left(x_{i}(t)\right)\right] \leq-\sum_{i=1}^{n} \sum_{k=1}^{n} \frac{k_{2}}{\bar{\omega}}\left|e_{i k}(t)\right| .
\end{aligned}
$$

Recalling controller (9) and combined with equations (10)-(15), based on the basic inequalities of Jensen's inequality, one can gain

$$
\begin{aligned}
\frac{\mathrm{d} V(t)}{\mathrm{d} t} \leq & -\left[\underline{\omega}+\frac{k_{1} \cdot \underline{\omega}}{\bar{\omega}}-\left(b^{\max }+c^{\max }+K^{\max }\right) \cdot n L^{\max }\right] \cdot \sum_{i=1}^{n} e_{i}^{T}(t) e_{i}(t) \\
& -\left[\frac{k_{2}}{\bar{\omega}}-\left(b^{\max }+c^{\max }+K^{\max }\right) \cdot n h^{\max }\right] \cdot \sum_{i=1}^{n} \sum_{k=1}^{n}\left|e_{i k}(t)\right| \\
\leq & -\kappa_{1} V(t)-\kappa_{2} V^{\frac{1}{2}}(t) .
\end{aligned}
$$

Then, according to assumption (H4) in this theorem, one can see $\kappa_{1}>0$ and $\kappa_{2}>0$, then we know that the origin of error system (6) is finite-time stable with feedback controller (7), and the settling time is obtained by

$$
t_{1}^{*} \leq \frac{2}{\kappa_{1}} \ln \frac{\kappa_{1} V^{\frac{1}{2}}(0)+\kappa_{2}}{\kappa_{2}}=\frac{2}{\kappa_{1}} \ln \frac{\kappa_{1}\|e(0)\|_{2}+\kappa_{2}}{\kappa_{2}} .
$$

Theorem 3.2 If conditions ( $\mathrm{H} 1)-(\mathrm{H} 3)$ are supported, then the response system (4) with adaptive controller (8) can synchronize to the corresponding drive system (1).

Proof In this proof, we let the new Lyapunov functional be as follows:

$$
\begin{aligned}
V(t)= & \frac{1}{2} \sum_{i=1}^{n} e_{i}^{T}(t) e_{i}(t)+\frac{n}{2} \cdot L^{\max } c^{\max } \int_{t-\tau}^{t} e^{T}(s) \cdot e(s) \mathrm{d} s \\
& +\sum_{i=1}^{n} \frac{1}{2 \rho_{i k}}\left(p_{i}-\theta\right)^{2}+\sum_{i=1}^{n} \frac{1}{2 \varrho_{i}}\left(q_{i}-\vartheta\right)^{2},
\end{aligned}
$$

where $\theta$ and $\vartheta$ are positive constants to be determined.

The derivative of Lyapunov function $V(t)$ can be obtained along the error system (6) as follows:

$$
\begin{aligned}
\dot{V}(t)= & \sum_{i=1}^{n} e_{i}^{T}(t)\left[-\left(a_{i}\left(\Phi_{i}\left(y_{i}(t)\right)\right)-a_{i}\left(\Phi_{i}\left(x_{i}(t)\right)\right)\right)+\sum_{j=1}^{n} b_{i j}(t) \gamma_{j}^{*}(t)+\sum_{j=1}^{n} c_{i j}(t) \gamma_{j}^{*}(t-\tau)\right. \\
& +\sum_{j=1}^{n} \int_{0}^{+\infty} K_{i j}(t, s) \gamma_{j}^{*}(s) \mathrm{d} s-\frac{p_{i}}{\varpi_{i}\left(y_{i}(t)\right)}\left(\pi_{i}(t)-\xi_{i}(t)\right) \\
& \left.-\frac{q_{i}}{\varpi_{i}\left(y_{i}(t)\right)} \operatorname{sign}\left(\pi_{i}(t)-\xi_{i}(t)\right)\right]
\end{aligned}
$$




$$
\begin{aligned}
& +\sum_{i=1}^{n}\left(p_{i}-\theta\right) e_{i}^{T}(t) \frac{1}{\varpi_{i}\left(y_{i}(t)\right)}\left(\pi_{i}(t)-\xi_{i}(t)\right) \\
& +\sum_{i=1}^{n}\left(q_{i}-\vartheta\right) e_{i}^{T}(t) \frac{1}{\varpi_{i}\left(y_{i}(t)\right)} \operatorname{sign}\left(\pi_{i}(t)-\xi_{i}(t)\right) \\
& +\frac{n}{2} \cdot L^{\max } c^{\max } e^{T}(t) e(t)-\frac{n}{2} \cdot L^{\max } c^{\max } e^{T}(t-\tau) e(t-\tau)
\end{aligned}
$$

Since

$$
\begin{aligned}
& -\sum_{i=1}^{n} e_{i}^{T}(t) \frac{\theta}{\varpi_{i}\left(y_{i}(t)\right)}\left(\Phi_{i}\left(y_{i}(t)\right)-\Phi_{i}\left(x_{i}(t)\right)\right) \leq-\sum_{i=1}^{n} \frac{\theta \cdot \underline{\omega}}{\bar{\omega}} e_{i}^{T}(t) e_{i}(t), \\
& -\sum_{i=1}^{n} e_{i}^{T}(t) \frac{\vartheta}{\varpi_{i}\left(y_{i}(t)\right)} \operatorname{sign}\left[\Phi_{i}\left(y_{i}(t)\right)-\Phi_{i}\left(x_{i}(t)\right)\right] \leq-\sum_{i=1}^{n} \sum_{k=1}^{n} \frac{\vartheta}{\bar{\omega}}\left|e_{i k}(t)\right| .
\end{aligned}
$$

It follows from (18)-(19), recalling (10)-(13), one can deduce that

$$
\begin{aligned}
\frac{\mathrm{d} V(t)}{\mathrm{d} t} \leq & -\left[\underline{\omega}+\frac{\theta \cdot \underline{\omega}}{\bar{\omega}}-\left(b^{\max }+c^{\max }+K^{\max }\right) \cdot n L^{\max }\right] \cdot \sum_{i=1}^{n} e_{i}^{T}(t) e_{i}(t) \\
& -\left[\frac{\vartheta}{\bar{\omega}}-\left(b^{\max }+c^{\max }+K^{\max }\right) \cdot n h^{\max }\right] \cdot \sum_{i=1}^{n} \sum_{k=1}^{n}\left|e_{i k}(t)\right| \\
\leq & -\kappa_{3} V(t)-\kappa_{4} V^{\frac{1}{2}}(t)
\end{aligned}
$$

Based on the definition of $\theta$ and $\vartheta$, we can choose suitable values of $\theta$ and $\vartheta$ to make $\kappa_{3}=$ $\underline{\omega}+\frac{\theta \cdot \underline{\omega}}{\bar{\omega}}-\left(b^{\max }+c^{\max }+K^{\max }\right) \cdot n L^{\max }>0$ and $\kappa_{4}=\frac{\vartheta}{\bar{\omega}}-\left(b^{\max }+c^{\max }+K^{\max }\right) \cdot n h^{\max }>0$. Then we prove that the origin of error system (6) is finite-time stable with adaptive controller (8), and the settling time is obtained by

$$
t_{1}^{*} \leq \frac{2}{\kappa_{3}} \ln \frac{\kappa_{3} V^{\frac{1}{2}}(0)+\kappa_{4}}{\kappa_{4}}=\frac{2}{\kappa_{3}} \ln \frac{\kappa_{3}\|e(0)\|_{2}+\kappa_{4}}{\kappa_{4}} .
$$

Remark 3.3 This paper considers that the distributed delays are unbounded, which is more difficult to verify than the bounded case. In the pervious results, the delay kernels satisfy

$$
K_{i j}= \begin{cases}1, & 0 \leq t \leq \tau_{i j} \\ 0, & t>\tau_{i j}\end{cases}
$$

where $\tau_{i j}>0$ are constants, then the CGNNs can be rewritten as a special case in this paper.

Remark 3.4 In fact, the finite time synchronization problem is very complex and difficult to calculate when there exist the discontinuity phenomenon, mixed delays, and switching controllers in the traditional neural network model. This paper overcomes these difficulties and has some innovation. By using Theorem 3.1 and Theorem 3.2, the finite-time synchronization problem can be generalized, that is, by choosing the appropriate controller, 
the stability time of the synchronization error system can be estimated more easily. On the other hand, the controller selected in this paper is more widely used as the estimation of stability time. It provides a theoretical basis for solving complex problems in engineering application.

\section{Examples}

Example 4.1 Consider the following two-dimensional discontinuous CGNNs with and mixed delays:

$$
\begin{aligned}
\frac{\mathrm{d} \pi_{i}(t)}{\mathrm{d} t}= & -\varpi_{i}\left(\pi_{i}(t)\right)\left[a_{i}\left(\pi_{i}(t)\right)-\sum_{j=1}^{n} b_{i j}(t) f_{j}\left(\pi_{j}(t)\right)-\sum_{j=1}^{n} c_{i j}(t) f_{j}\left(\pi_{j}\left(t-\tau_{i j}(t)\right)\right)\right. \\
& \left.-\sum_{j=1}^{n} \int_{0}^{+\infty} K_{i j}(t, s) f_{j}\left(\pi_{j}(s)\right) \mathrm{d} s-I_{i}(t)\right], \quad i=1,2 .
\end{aligned}
$$

Let $\varpi_{1}\left(\pi_{1}(t)\right)=0.5+0.1 \cos \left(\pi_{1}(t)\right), \varpi_{2}\left(\pi_{2}(t)\right)=0.5-0.1 \sin \left(\pi_{2}(t)\right), a_{1}\left(\pi_{1}(t)\right)=-0.4 \pi_{1}(t)$, $a_{2}\left(\pi_{2}(t)\right)=-0.4 \pi_{2}(t), b_{11}(t)=b_{22}(t)=0.1, b_{12}(t)=b_{21}(t)=0, c_{11}(t)=c_{22}(t)=0.2, c_{12}(t)=$ $c_{21}(t)=0, K_{11}=K_{12}=K_{21}=K_{22}=1$, and $I_{1}(t)=0.2 \sin \sqrt{2} t+0.1 \sin \sqrt{5} t, I_{2}(t)=0.3 \times$ $\cos \sqrt{3} t-0.2 \sin 2 t$.

The two neuron activation functions which satisfy the two conditions $(\mathrm{H} 1)-(\mathrm{H} 2)$ are designed as follows:

$$
f_{1}(\cdot)=f_{2}(\cdot)= \begin{cases}x-0.1, & x<0 \\ x+0.1, & x \geq 0\end{cases}
$$

Let $L^{\max }=h^{\max }=1$, then we consider the control rule $u_{i}(t)=-k_{1}\left(\pi_{i}(t)-\xi(t)\right)-$ $k_{2} \operatorname{sign}\left(\pi_{i}(t)-\xi(t)\right)$ with $k_{1}=k_{2}=3.5$. It is easy to check that

$$
\begin{aligned}
& 2.7=\underline{\omega}+\frac{k_{1} \cdot \underline{\omega}}{\bar{\omega}}>\left(b^{\max }+c^{\max }+K^{\max }\right) \cdot n L^{\max }=2.6, \\
& 5.8=\frac{k_{2}}{\bar{\omega}}>\left(b^{\max }+c^{\max }+K^{\max }\right) \cdot n h^{\max }=2.6,
\end{aligned}
$$

which show that the assumption in Theorem 3.1 is satisfied. Figure 1 and Fig. 2(c) indicate the simulation results.

Example 4.2 Consider the following discontinuous CGNNs with mixed delays:

$$
\begin{aligned}
\frac{\mathrm{d} \pi_{i}(t)}{\mathrm{d} t}= & -\varpi_{i}\left(\pi_{i}(t)\right)\left[a_{i}\left(\pi_{i}(t)\right)-\sum_{j=1}^{n} b_{i j}(t) f_{j}\left(\pi_{j}(t)\right)-\sum_{j=1}^{n} c_{i j}(t) f_{j}\left(\pi_{j}\left(t-\tau_{i j}(t)\right)\right)\right. \\
& \left.-\sum_{j=1}^{n} \int_{0}^{+\infty} K_{i j}(t, s) f_{j}\left(\pi_{j}(s)\right) \mathrm{d} s-I_{i}(t)\right], \quad i=1,2,
\end{aligned}
$$

where the parameters have the same meanings as in equation (21).

We consider the novel adaptive controller $u_{i}(t)=-p_{i}\left(\pi_{i}(t)-\xi(t)\right)-q_{i} \operatorname{sign}\left(\pi_{i}(t)-\xi(t)\right)$ with $\dot{p}_{i}=e_{i}^{T}(t) \frac{\varrho_{i}}{\varpi_{i}\left(y_{i}(t)\right)}\left(\pi_{i}(t)-\xi_{i}(t)\right), \dot{q}_{i}=e_{i}^{T}(t) \frac{\varrho_{i}}{\varpi_{i}\left(y_{i}(t)\right)} \operatorname{sign}\left(\pi_{i}(t)-\xi_{i}(t)\right)$. For CGNNs (22), we 


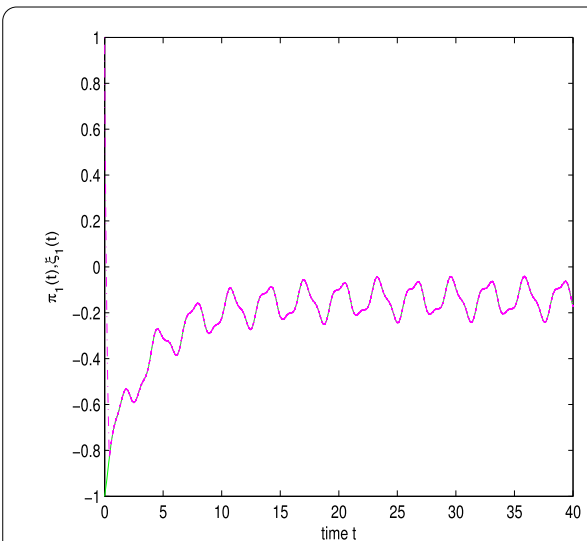

(a)

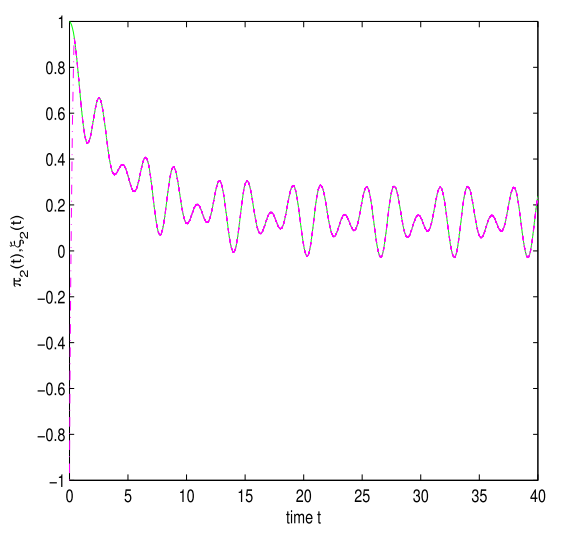

(b)

Figure $1(\mathbf{a})$ and $(\mathbf{b})$ show the state trajectories $\pi_{1}(t), \xi_{1}(t)$ and state trajectories $\pi_{2}(t), \xi_{2}(t)$ in Example 4.1 and Example 4.2, respectively

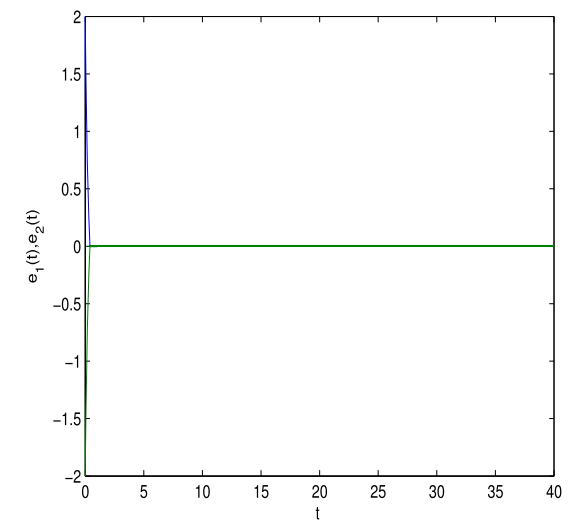

(c)

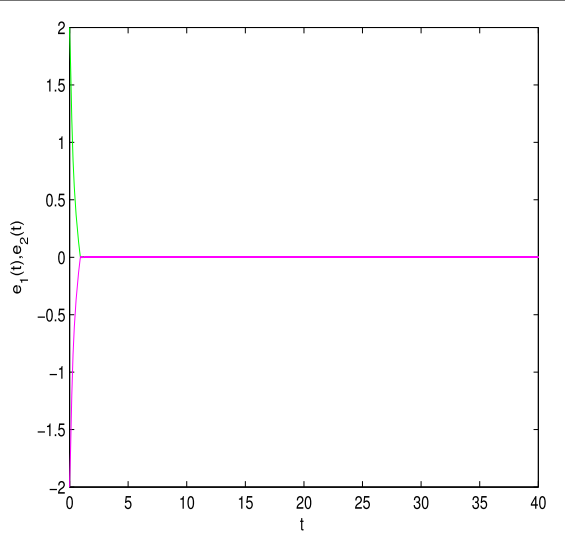

$(d)$

Figure 2 (c) Synchronization error trajectories of the drive system and the response system with feedback controller can be achieved in finite-time in Example 4.1; (d) Synchronization error trajectories of the drive system and the response system with adaptive controller can be achieved in finite-time in Example 4.2

can choose suitable parameters $\theta=3$ and $\vartheta=3$ to make the condition in Theorem 3.2 be satisfied. With given random initial state, Fig. 2(d) shows that the two state trajectories approach the zero solution, then the simulation result is presented to illustrate the obtained theoretical findings.

\section{Conclusions}

The finite-time synchronization problem of discontinuous CGNNs with mixed delay is studied in this brief. By using the Lyapunov functional framework, new mathematical analysis techniques, Filippov theory, and inequality techniques, a new state feedback controller and an adaptive controller are constructed to realize finite-time synchronization of complex neural networks. Compared with previous results, we overcome the problem of non-Lipschitz continuity system, so how to deal with the right discontinuous system is a challenge. Finally, two numerical simulations verify the advantages of the proposed switching control method and mathematical calculation method. Future research will fo- 
cus on the analysis of neural networks with time delay and the design of more effective coupling schemes between different neurons in the system.

\author{
Acknowledgements \\ Not applicable. \\ Funding \\ This work is supported by the National Natural Science Foundation of China (11801042) and Training Program for \\ Excellent Young Innovators of Changsha (kq2009023).
}

Availability of data and materials

Data sharing allows researchers to verify the results of an article, replicate the analysis, and conduct secondary analyses.

Competing interests

The authors declare that they have no competing interests.

\title{
Authors' contributions
}

ZX and RL initiated and discussed the research problem; ZX verified the correctness of the experimental results; $R L$ performed the experiments and took figures, analyzed the data; ZX drafted the paper. All authors have read and approved the paper.

\section{Author details}

${ }^{1}$ Department of Mathematics and Computer Science, Changsha University, Changsha 410022, China. ${ }^{2}$ Science and

Technology Management Services of Hunan Torch Entrepreneurship Center, Changsha 410000, China.

\section{Publisher's Note}

Springer Nature remains neutral with regard to jurisdictional claims in published maps and institutional affiliations.

Received: 19 January 2021 Accepted: 4 August 2021 Published online: 26 August 2021

References

1. Cortes, J.: Discontinuous dynamical systems. IEEE Control Syst. Mag. 28(3), 36-73 (2008)

2. Akhmet, M.: Principles of discontinuous dynamical systems. Plant Physiol. 146(3), 1064-1074 (2010)

3. Heemels, W., Weiland, S.: Input-to-state stability and interconnections of discontinuous dynamical systems. Automatica 44(12), 3079-3086 (2008)

4. Michel, A., Sun, Y., Molchanov, A.: Stability analysis of discontinuous dynamical systems determined by semigroups. IEEE Trans. Autom. Control 50(9), 1277-1290 (2005)

5. Cao, J., Wang, Z., Sun, Y.: Synchronization in an array of linearly stochastically coupled networks with time delays. Phys. A, Stat. Mech. Appl. 385(2), 718-728 (2007)

6. Yang, X., Cao, J., Lu, J.: Stochastic synchronization of coupled neural networks with intermittent control. Phys. Lett. A 373(36), 3259-3272 (2009)

7. Song, Q.: Synchronization analysis of coupled connected neural networks with mixed time delays. Neurocomputing 72(16-18), 3907-3914 (2009)

8. Cai, Z., Huang, L., Zhang, L.: Finite-time synchronization of master-slave neural networks with time-delays and discontinuous activations. Appl. Math. Model. 47, 208-226 (2017)

9. Cohen, M., Grossberg, S.: Absolute stability of global pattern formation and parallel memory storage by competitive neural networks. IEEE Trans. Syst. Man Cybern. 13(5), 815-826 (1983)

10. Wang, Z., Liu, Y., Li, M., et al.: Stability analysis for stochastic Cohen-Grossberg neural networks with mixed time delays. IEEE Trans. Neural Netw. 17(3), 814-820 (2006)

11. Wang, D., Huang, L.: Almost periodic dynamical behaviors for generalized Cohen-Grossberg neural networks with discontinuous activations via differential inclusions. Commun. Nonlinear Sci. Numer. Simul. 19(10), 3857-3879 (2014)

12. Wang, D., Huang, L.: Periodicity and global exponential stability of generalized Cohen-Grossberg neural networks with discontinuous activations and mixed delays. Neural Netw. 51(3), 80-95 (2014)

13. Wang, J., Tian, L., Global, Z.Z.: Lagrange stability for Takagi-Sugeno fuzzy Cohen-Grossberg BAM neural networks with time-varying delays. Int. J. Control. Autom. Syst. 16(4), 1603-1614 (2018)

14. Ravi, A., Snezhana, H., O'Regan, D., et al.: Stability analysis of Cohen-Grossberg neural networks with random impulses. Mathematics 6(9), 144-156 (2018)

15. Feng, J., Xu, S.: New criteria on global robust stability of Cohen-Grossberg neural networks with time-varying delays. Neurocomputing 72(1-3), 445-457 (2008)

16. Kong, F., Rakkiyappan, R.: Finite-time and fixed-time synchronization control of discontinuous fuzzy Cohen-Grossberg neural networks with uncertain external perturbations and mixed time delays. Fuzzy Sets Syst. 411, 105-135 (2021)

17. Kong, F., Zhu, Q., Sakthivel, R.: Finite-time and fixed-time synchronization control of fuzzy Cohen-Grossberg neural networks. Fuzzy Sets Syst. 394, 87-109 (2020)

18. Zhang, Z., Yu, S.: Global asymptotic stability for a class of complex-valued Cohen-Grossberg neural networks with time delays. Neurocomputing 171, 1158-1166 (2016)

19. Zhang, X., Li, C., Huang, T.: Impacts of state-dependent impulses on the stability of switching Cohen-Grossberg neural networks. Adv. Differ. Equ. 2017(1), 316 (2017)

20. Hu, J., Zeng, C.: Adaptive exponential synchronization of complex-valued Cohen-Grossberg neural networks with known and unknown parameters. Neural Netw. 86, 90-101 (2016) 
21. Abdurahman, A., Jiang, H., Teng, Z.: Lag synchronization for Cohen-Grossberg neural networks with mixed time-delays via periodically intermittent control. Int. J. Comput. Math. 94, 275-295 (2017)

22. Filippov, A.: Differential Equations with Discontinuous Right-Hand Side. Mathematics and Its Applications (Soviet Series). Kluwer Academic, Boston (1988)

23. Forti, M., Nistri, P.: Global convergence of neural networks with discontinuous neuron activations. IEEE Trans. Circuits Syst. I, Fundam. Theory Appl. 50(11), 1421-1435 (2003)

24. Forti, M., Nistri, P., Quincampoix, M.: Generalized neural network for nonsmooth nonlinear programming problems. IEEE Trans. Circuits Syst. I, Regul. Pap. 51(9), 1741-1754 (2004)

25. Kong, F., Zhu, Q., Huang, T.: New fixed-time stability lemmas and applications to the discontinuous fuzzy inertial neural networks. IEEE Trans. Fuzzy Syst. (2020). https://doi.org/10.1109/TFUZZ.2020.3026030

26. Yang, X., Wu, Z., Cao, J.: Finite-time synchronization of complex networks with nonidentical discontinuous nodes. Nonlinear Dyn. 73(4), 2313-2327 (2013)

27. Yang, X:: Can neural networks with arbitrary delays be finite-timely synchronized? Neurocomputing 143(16), 275-28 (2014)

28. Chandrasekar, A., Rakkiyappan, R., Cao, J., et al.: Synchronization of memristor-based recurrent neural networks with two delay components based on second-order reciprocally convex approach. Neural Netw. 57, 79-93 (2014)

29. Jiang, C., Zhang, F., Li, T.: Synchronization and anti-synchronization of $\mathrm{N}$-coupled fractional-order complex chaotic systems with ring connection. Math. Methods Appl. Sci. 47(7), 2625-2638 (2018)

30. Ahmed, M., Liu, Y., Zhang, W., et al.: Exponential synchronization via pinning adaptive control for complex networks of networks with time delays. Neurocomputing 225(C), 198-204 (2017)

31. Huang, X., Lam, J., Cao, J., et al.: Robust synchronization criteria for recurrent neural networks via linear feedback. Int. J. Bifurc. Chaos 17(8), 2723-2738 (2007)

32. Xiong, W., Takougang, K., Christos, V., et al.: A fractional system with five terms: analysis, circuit, chaos control and synchronization. Int. J. Electron. 106(1), 109-120 (2018)

33. Yang, X., Ho, D., Lu, J., et al.: Finite-time cluster synchronization of T-S fuzzy complex networks with discontinuous subsystems and random coupling delays. IEEE Trans. Fuzzy Syst. 23(6), 2302-2316 (2015)

34. Yang, X., Song, Q., Liang, J., et al.: Finite-time synchronization of coupled discontinuous neural networks with mixed delays and nonidentical perturbations. J. Franklin Inst. 352(10), 4382-4406 (2015)

35. Peng, D., Li, X., Aouiti, C., et al.: Finite-time synchronization for Cohen-Grossberg neural networks with mixed time-delays. Neurocomputing 294, 39-47 (2018)

36. Yang, C., Huang, L.: Finite-time synchronization of coupled time-delayed neural networks with discontinuous activations. Neurocomputing 249(8), 64-71 (2017)

37. Zhang, C., Wang, X., Wang, S., et al.: Finite-time synchronization for a class of fully complex-valued networks with coupling delay. IEEE Access 6, 17923-17932 (2018)

38. Zheng, M., Li, L., Peng, H., et al.: Finite-time stability and synchronization of memristor-based fractional-order fuzzy cellular neural networks. Commun. Nonlinear Sci. Numer. Simul. 59, 272-291 (2018)

39. Aubin, J., Cellina, A.: Differential Inclusions, pp. 8-13. Springer, Berlin (1984)

40. Hardy, G., Littlewood, J.: Inequalities. Cambridge University Press, London (1988)

41. LaSalle, J.: The Stability of Dynamical Systems. SIAM, Philadelphia (1976)

42. Clarke, F:: Optimization and Nonsmooth Analysis. SIAM, Philadelphia (1990)

43. Polyakov, A.: Nonlinear feedback design for fixed-time stabilization of linear control systems. IEEE Trans. Autom. Control 57(8), 2106-2110 (2012)

44. Strogatz, S., Stewart, l.: Coupled oscillators and biological synchronization. Sci. Am. 269(6), 102-109 (1993)

45. Milanovi, V., Zaghloul, M.: Synchronization of chaotic neural networks and applications to communications. Int. J. Bifurc. Chaos 6(12b), 2571-2585 (1996)

\section{Submit your manuscript to a SpringerOpen ${ }^{\circ}$ journal and benefit from:}

- Convenient online submission

- Rigorous peer review

- Open access: articles freely available online

- High visibility within the field

- Retaining the copyright to your article

Submit your next manuscript at $\gg$ springeropen.com 